\title{
A Method of Dual-Sensor Signal Fusion for DSP-Based Wide-Range Vibration Detection and Control
}

\author{
Huaizhong Li \\ Griffith School of Engineering, Gold Coast Campus, Griffith University, QLD 4222, \\ Australia \\ Phone: +61 (7) 555 28252; Telefax: +61 (7) 555 28065; \\ E-mail: lihuaizhong@gmail.com; h.li@griffith.edu.au
}

\begin{abstract}
This paper presents a unique dual-sensor signal fusion technology for DSP (digital signal processor)-based wide range vibration detection and active vibration control (AVC), which aims to suppress wide range vibration disturbances up to sub-micron level. In this method, two accelerometer sensors with different measurement ranges and sensitivities are used to detect the vibration disturbances as coarse and fine sensors respectively, and feed the signals simultaneously to a DSP controller. Each sensor is responsible for detecting accelerations in a specific range. By proper incorporation of signals from the two sensors, it is possible to achieve a wide detection range of vibrations at low cost. Simulation study shows that in an AVC system with the proposed dual-sensor signal fusion approach as the vibration detection component, significant improvement can be achieved comparing with traditional singlesensor AVC systems.
\end{abstract}

Keywords: Active vibration control; Vibration detection; Digitization; Sensor fusion; Accelerometer; DSP controller. 


\section{Introduction}

Vibration control becomes increasingly important for high-precision equipment developers to respond to the request for more stable processes and higher positioning accuracies. Due to the limitations of passive vibration control approaches, considerable attention has been paid to active vibration control systems. An active vibration control (AVC) system continuously senses and reacts to vibration disturbances. It uses actuators, sensors and electronic control to reduce vibrations in real-time. Vibration is attacked with a counter-force that drives the structure to respond in a way that the total response of the system at the location of interest can then be significantly reduced $[1,2]$. A schematic of the control loop for a typical AVC system is shown in figure 1 . There is an increasing demand for innovative active vibration control systems that can provide wide vibration suppression range up to sub-micron level or even nanometre level. An economic and efficient vibration detection approach is needed in such an AVC system.

The state-of-the-art active systems employ digital signal processors (DSPs) as the embedded controller. Comparing with an analogue controller, a DSP is designed for very-high-speed numeric real-time processing of digitized signals, and is generally considered as well suited for most modern control systems. It can provide straightforward adjustment of control parameters, easy incorporation of adaptation, estimation and identification stages, and direct overall operational monitoring and maintenance $[3,4]$.

The first step in AVC is the detection of vibration disturbances. Although direct velocity feedback is ideal for active vibration control [2], it is difficult to measure the vibration velocity due to the lack of a reference position for a sensor to measure the absolute velocity, and also the extensive cost for the velocity sensor like laser Doppler vibrometer (LDV). 
Therefore most AVC systems are using accelerometers as the feedback sensor because of the low cost, small size and easy to use [5-7].

A problem with a DSP-based AVC system using acceleration feedback is to deal with a wide dynamic range of the vibration disturbances. The acceleration signal from a common accelerometer device such as a piezoelectric accelerometer is an analogue signal. To prepare the acceleration signals for processing by digital hardware like a DSP controller, it has to be converted to digital signals by an analogue-to-digital converter (ADC). Analogue-to-digital conversion, also known as digitization, consists of the sampling (digitization in time) and quantization (digitization in amplitude) processes. In the quantization process, the amplitude of each discrete-time sample is quantized into one of the $2^{B}$ levels, where $B$ is the number of bits that the ADC has to represent for each sample [4]. Due to the fact of finite number of bits, there exists a resolution given by dividing a full-scale range with the number of quantization levels, $2^{B}$, and the difference between the quantized number and the original value is defined as the quantization error or noise [4]. It is clear that an ADC with higher number of bits can output more quantization levels, and thus can represent analogue signals more accurately with larger signal-to-quantization-noise ratio (SQNR). However, high number of bits means high cost, which is controversial to the requirements of the embedded control systems on compact design, low power, and low cost. Therefore a trade-off between the measurement range and accuracy is necessary. For the most widely used DSP controllers for motor control or vibration control, such as the TI C2000 platforms, the integrated A/D converters are 12-bit. A 12-bit ADC has $4096\left(2^{12}\right)$ levels of quantization, which provides 72 dB SQNR.

The dynamic range of vibration signals is usually very large in reality. For a sinusoidal vibration signal, the amplitude in terms of displacement $D$ and acceleration $A$ can be 
correlated by $A=\omega^{2} D$, where $\omega=2 \pi f$ is the angular frequency of vibration. For vibration with sub-micron level displacement $\left(D<10^{-6} \mathrm{~m}\right)$ at low vibratory frequency ( $f$ in the range of a few Hertz), the resultant acceleration level $(A)$ to be detected is very small. On the other hand, vibration with high level displacement at high frequency can exhibit very high acceleration. Thus it is highly desirable for the AVC system to be able to detect vibration at a wide dynamic rang in terms of acceleration with acceptable accuracy.

However, this is hard to realize in practice with a single sensor system due to two reasons. Firstly, as a result of hardware limitation, accelerometers with high upper detection limits usually have big threshold values for the lower limits. On the other hand, those with small threshold values can only detect accelerations within a small range. For example, a PCB model 352C03 accelerometer has a measurement range of $\pm 500 \mathrm{~g}$ pk and a sensitivity of 10 $\mathrm{mV} / \mathrm{g}$, with a broadband resolution of $0.0005 \mathrm{~g}$ rms. In comparison, a PCB model 352C33 accelerometer has a measurement range of $\pm 50 \mathrm{~g}$ pk and a sensitivity of $100 \mathrm{mV} / \mathrm{g}$, with a broadband resolution of $0.00015 \mathrm{~g}$ rms. Thus it is difficult to find a single sensor that can satisfy acceleration detection needs on both the high measurement range and also good resolution. Secondly, the ADC unit for a DSP controller has a limited input voltage range and a limited bit-resolution for its converted digital results. There always exists a dilemma between extending the upper and lower limits of the acceleration detection range with singlesensor system.

In view of the problems associated with current DSP-based active vibration control system using acceleration feedback, a method of dual-sensor signal fusion for wide-range vibration detection and control is introduced in this paper, which can effectively extend both the upper and lower detection limits thus realize wide-range acceleration detection. 


\section{Methodology}

\subsection{Detection with single sensor}

Suppose that an $n$-bit ADC unit in a DSP controller is used with input voltage range from $V_{i n \min }$ to $V_{i n \max }$, it can convert up to $2^{n}$ levels of digital results with each level corresponding to an input voltage step of $\frac{V_{i n \max }-V_{i n \min }}{2^{n}}$.

By employing a suitable signal conditioning circuit for amplification and level shifting, the analogue signal from an accelerometer can be adjusted to match the ADC input range such that the ADC voltage limits $V_{i n \min }$ and $V_{i n \max }$ can map the accelerations limits $A_{\min }$ and $A_{\max }$, and the voltage step $\frac{V_{i n \max }-V_{\text {inmin }}}{2^{n}}$ corresponds to acceleration step $\frac{A_{\max }-A_{\min }}{2^{n}}$. Thus for a single sensor system, the detectable acceleration amplitude range is from $\frac{A_{\max }-A_{\min }}{2^{n}}$ to $A_{\max }$ , as illustrated in Figure 2. If the acquired ADC output at a time instant $t$ is $p$, where $p$ is an integer and $0<=p<=2^{n}-1$, then the actual vibration acceleration is

$q(t)=g\left(p(t)-p_{0}\right)$

Where $p_{0}$ is a level-shifting constant which is corresponding to the ADC output value for a zero vibration acceleration value, $g$ is a gain coefficient. For piezoelectric accelerometers, $A_{\max }=-A_{\min }$, thus Eq. (1) can be further expressed as

$$
q(t)=\left(p(t)-2^{n-1}\right)\left(\frac{A_{\max }-A_{\min }}{2^{n}}\right) .
$$

To detect wide range of accelerations, it is necessary to minimize the lower detection limit $\frac{A_{\max }-A_{\min }}{2^{n}}$ and maximize the upper limit $A_{\max }$ (thus maximize $A_{\max }-A_{\min }$ ). Clearly, it is 
impossible to achieve both at the same time by using DSP-based vibration detection from a single accelerometer and an $n$-bit ADC unit. The single-sensor feedback AVC system has the problems of limited vibration range or limited resolution thus cannot achieve wide range vibration control up to sub-micron level or nanometer level.

\subsection{Dual-sensor approach}

To solve the aforementioned problems with single-sensor feedback AVC system, a dualsensor method making use of two ADC channels of the DSP controller is designed. Two accelerometers with different measurement ranges and sensitivities are used to detect the vibration disturbances as coarse and fine sensors respectively, and feed the signals simultaneously to the DSP controller.

Assume that the same $n$-bit ADC units are used with an input voltage range from $V_{i n \min }$ to $V_{i n \max }$. Sensor 1 with small measurement range $\left(A_{1 \min }\right.$ to $\left.A_{1 \max }\right)$ and high sensitivity serves as fine input to ADC channel 1 , and Sensor 2 with high measurement range $\left(A_{2 \min }\right.$ to $\left.A_{2 \max }\right)$ and low sensitivity serves as coarse input to ADC channel 2. By properly adjusting the offset and gain of individual signal conditioning circuits, the acceleration limits $A_{1 \max }$ and $A_{2 \max }$ can be adjusted to match with $V_{i n \max }, A_{1 \min }$ and $A_{2 \min }$ with $V_{\text {inmin }}$, respectively. Thus for the same input voltage step $\frac{V_{i n \max }-V_{i n \min }}{2^{n}}$, the corresponding acceleration steps for channel 1 and channel 2 are different. For channel 1 , the step is $\frac{A_{1 \max }-A_{1 \min }}{2^{n}}$, and for channel 2 it is $\frac{A_{2 \max }-A_{2 \min }}{2^{n}}$. Clearly $A_{2 \max }$ is much bigger than $A_{1 \max }$, hence $\frac{A_{2 \max }-A_{2 \min }}{2^{n}}$ is much larger than $\frac{A_{1 \max }-A_{1 \min }}{2^{n}}$, as illustrated in Figure 3. 
A key step is to combine the signals from the two sensors to extend the acceleration detection range to $A_{2 \max }$ with a minimum detection level of $\frac{A_{1 \max }-A_{1 \min }}{2^{n}}$. To implement this sensor fusion method, let the acquired ADC channel 1 output corresponding to the fine sensor at a time instant $t$ is $p_{1}$. If the ADC channel 1 is not saturated $\left(0<p_{1}(t)<2^{n}-1\right)$, it means that the vibration is within the measurement range of Sensor 1 , then Sensor 1 signal acquired by ADC channel 1 is used, which can be expressed as

$$
q(t)=\left(p_{1}(t)-2^{n-1}\right)\left(\frac{A_{1 \max }-A_{1 \min }}{2^{n}}\right)
$$

If the ADC channel 1 is saturated $\left(p_{1}(t)=0\right.$ or $\left.p_{1}(t)=2^{n}-1\right)$, it means that the current vibration is out of the measurement range for the fine sensor. Now Sensor 2 signal acquired by ADC channel 2 has to be used. Let the acquired ADC channel 2 output corresponding to the course sensor (Sensor 2) at a time instant $t$ is $p_{2}$. The detected vibration acceleration is

$$
q(t)=\left(p_{2}(t)-2^{n-1}\right)\left(\frac{A_{2 \max }-A_{2 \min }}{2^{n}}\right)
$$

A flowchart of the implementation algorithm is shown in Figure 4. Both channels of the ADC unit are sampled and converted at the same time. The digital result of ADC channel 1 is constantly used to check the saturation to determine which range the current acceleration sample belongs to and select the corresponding signal for further processing. With this signal selection algorithm, Sensor 1 signals are used for low level acceleration of $A_{1 \min }$ to $A_{1 \max }$, while Sensor 2 signals are used for high level accelerations beyond the measurement range of Sensor 1. Wide range acceleration detection can be achieved using this dual-sensor system. 


\section{Results and discussions}

To assess the functionality of the proposed dual-sensor detection approach and its effectiveness in AVC system, a series of simulation study is carried out using Matlab/Simulink models. The specifications of the two accelerometers, Sensor 1 and Sensor 2, are listed in Table 1 . With a higher sensitivity, Sensor 1 is selected for fine level vibration detection, while Sensor 2 with a high measurement range and low sensitivity is responsible for high level vibrations.

By properly choosing the voltage gains and biases of the two individual signal conditioning circuits, the effective output from the conditioning circuits of Sensor 1 can be set as $A_{1 \max }=0.1 \mathrm{~g} \cong 1 \mathrm{~m} / \mathrm{s}^{2}$, and $A_{1 \min }=-0.1 \mathrm{~g} \cong-1 \mathrm{~m} / \mathrm{s}^{2}$. The resultant $A_{1 \min }$ and $A_{1 \max }$ are smaller than the actual measurement range for Sensor 1, this is to achieve a better measurement resolution for small vibration levels. For Sensor 2, the full measurement range is used which has $A_{2 \max } \cong 500 \mathrm{~m} / \mathrm{s}^{2}$, and $A_{2 \min } \cong-500 \mathrm{~m} / \mathrm{s}^{2}$. In a DSP controller, 2 channels of 12-bit ADC unit with an input voltage range from $0 \mathrm{~V}$ to $3 \mathrm{~V}$ are used and the sampling rate is set to be $18.31 \mathrm{kHz}$, i.e., sampling time $T_{s}=5.5 \times 10^{-5} \mathrm{~s}, n=12, V_{\text {inmin }}=0 \mathrm{~V}$ and $V_{\text {inmax }}=3 \mathrm{~V}$. There are $2^{n}=2^{12}=4096$ levels of the quantization result, and each level corresponds to a quantization step size of 3V/4096 $=0.000732 \mathrm{~V}$. Hence the dynamic range of accelerations this dual-sensor system can detect is from $\frac{A_{1 \max }-A_{1 \min }}{2^{n}}=\frac{2}{4096}=4.88 \times 10^{-4} \mathrm{~m} / \mathrm{s}^{2}$ to $A_{2 \max }=500 \mathrm{~m} / \mathrm{s}^{2}$.

A SIMULINK model of the dual sensor detection system is shown in Figure 5. In the model, a simple logic switch is used to simulate the signal selection algorithm. In order to demonstrate the signal fusion effect of this approach, a sinusoidal acceleration signal with amplitude of $3 \mathrm{~m} / \mathrm{s}^{2}$ is generated and applied to the detection component, and the output signal is displayed by an oscilloscope shown in Figure 6. 
Using the selection algorithm for dual-sensor fusion, the acquired digital acceleration signal comprises of two parts. From $0 \mathrm{~m} / \mathrm{s}^{2}$ to $1 \mathrm{~m} / \mathrm{s}^{2}\left(A_{1 \max }\right)$, the acceleration signal is quite smooth, this is the output from Sensor 1, which has a smaller step-size of $4.88 \times 10^{-4} \mathrm{~m} / \mathrm{s}^{2}$. From $1 \mathrm{~m} / \mathrm{s}^{2}$ to $3 \mathrm{~m} / \mathrm{s}^{2}$, clear signal steps are visible. This is the output from Sensor 2, which has a bigger step-size of $\frac{A_{2 \max }-A_{2 \min }}{2^{n}}=\frac{1000}{4096}=0.244 \mathrm{~m} / \mathrm{s}^{2}$. Clearly, Sensor 1 provides a high resolution for low level vibrations and Sensor 2 provides a wider detection range for high level vibrations. Effective signal fusion is achieved with this dual-sensor detection approach.

The dual-sensor detection approach is then integrated into an active vibration control system model as shown in Figure 7 to assess the effectiveness. A second-order dynamic system is used to model the plant vibrational behaviour. The natural frequency is set as $f_{n}=40 \mathrm{~Hz}$ (i.e., $\left.\omega_{n}=2 \pi f_{n}=251.2 \mathrm{rad}\right)$, and the damping ratio is $\zeta=1 \%$. The plant transfer function between force and acceleration can be expressed as

$$
Y(s)=\frac{s^{2}}{s^{2}+2 \zeta \omega_{n} s+\omega_{n}{ }^{2}}=\frac{s^{2}}{s^{2}+5.02{ }_{n} s+63101}
$$

An external step disturbing force is added to act on the plant with a magnitude of $500 \mathrm{~N}$. For comparison, four system models are constructed. The first model is an uncompensated plant. The second model as shown in Figure 8 is the active vibration control system equipped with the proposed dual-sensor detection component described above (with the same sensor specifications) for feedback. Velocity feedback is used as the control algorithm through an integral of the acquired acceleration signal. An integrator takes in the detected acceleration 
signal and calculates corresponding plant velocity. Feedback gain is set to 180 to simulate the compensating actuator force produced. To compare the differences between single- and dualsensor systems, models using only Sensor 1 or Sensor 2 for acceleration detection are also constructed. The same integrator and feedback gain are employed for both the single- and dual-sensor systems such that any performance difference comes from the detection component. The plant displacements of all four systems are monitored by an oscilloscope through a double integration component. The simulation is carried out for 5 seconds.

As discussed, Sensor 1 has a high sensitivity for low level vibrations but a small dynamic range; Sensor 2 has a high detection range but a low sensitivity. It is expected that there would be some problems with a single sensor feedback using either Sensor1 or Sensor 2. Sensor 1 only AVC would be good for low level vibration control but ineffective if the vibration level is high. Sensor 2 only AVC system would be effective for suppressing high level vibrations but unable to detect low level vibration. The dual-sensor feedback system would incorporate the merits of both sensors and perform more effectively in terms of vibration suppression at wider vibration levels.

Simulation results with the above four systems are presented in Figure 9, together with a zoomed view of the vibration responses shown in Figure 10. It can be seen in Figure 9(a) that with the external step disturbance, the uncompensated plant vibrates with a slow attenuation, resulting in a long settling time. The vibration amplitude is relatively large, starting from an amplitude of about $8 \times 10^{-3} \mathrm{~m}$, and decaying slowly. The vibration amplitude is still prominent with a value of about 0.5 micron after 5 second.

Single sensor feedback control using only Sensor 1 is proved to be quite ineffective for the initial stage when the vibration level is high. From the waveforms shown in Figure 9(b), the 
plant vibration amplitude is even higher than that from the uncompensated plant as shown in Figure 9(a). This is because that the sensor is saturated at this stage with the high vibration level, and the compensation force from the AVC system works as another disturbance rather than to counteract the vibration. However, when the vibration amplitude decreases to a lower level within the measurement range of the sensor, the feedback control system starts to work well, as demonstrated bythe zoomed view shown in Figure 10(b).

As to the single sensor AVC using only Sensor 2, it works quite well in suppressing the high level vibrations at the initial stage. As can be seen from Figure 9(c), the large amplitude vibration is quickly suppressed. However, the system is ineffective in control the low level vibrations as the sensor system does not have a good enough resolution to detect the small vibration disturbance. It can be seen from the zoomed view in Figure 10(c) that low level vibration with an amplitude of about 0.5 micron is quite clear at 1.4 second and lasts for long.

The proposed dual-sensor feedback control system gives the best performance among the four models tested, as shown in Figure 9(d). It provides a quick and effective suppression for the high level vibrations at the initial stage, benefiting from the high upper detection limit of Sensor 2. Further, it also works well in the low vibration range, thanks to the fine sensor with a better detection resolution. A zoomed view of the plant vibration in Figure 10(d) shows that vibration is well suppressed after 1.15 second. It demonstrates that sub-micron level vibration control can be essentially achieved with the dual-sensor approach. The detection component can be developed into a wide-range acceleration sensing system to replace the traditional single-accelerometer detection system. By incorporating signal detection from the two sensors with difference sensitivity and measurement range, the new approach achieves a wider detection range and higher accuracy for fine vibration than from single-sensor systems. Implementation of this dual-sensor approach in a feedback loop of a DSP-based active 
vibration control system can produce high-precision active vibration suppression up to submicron level or even better.

\section{Conclusions}

This paper introduced a novel method of dual-sensor signal fusion for wide-range vibration detection and control, which aims to solve the problems associated with DSP-based active vibration control system using acceleration feedback with a single sensor. The proposed approach is mainly for the application of active vibration control where cheap and efficient vibration detection is needed. By employing two sensors with different sensitivity and measurement range, this approach provides wide-range acceleration detection with better resolution when the vibration level is small. Each sensor is responsible for detecting accelerations in a specific range. With the help of the signal fusion algorithm, sensor signals are effectively combined and the overall performance is significantly improved, which enables effective active vibration control up to sub-micron level.

\section{Acknowledgement}

The author would like to thank Tong Lin from Nanyang Technological University (NTU) in Singapore for his contribution in this study, and also to thank Singapore Institute of Manufacturing Technology (SIMTech) for the support.

\section{References}

[1].Li, H.Z., Lin, W.J., and Yang, G.L., "Development of an Active Vibration Isolation System for Precision Machines”, Proceedings of SPIE, Volume 7522, 2010. 4th International Conference on Experimental Mechanics (ICEM2009), 18-20 Nov. 2009, Singapore (2009) 
[2].Preumont, A., Vibration control of active structures: an introduction. 3rd ed. Solid mechanics and its applications. 2011, Berlin: Springer. xx, 432 p.

[3].L. Dozio, P. Mantegazza, General-purpose processors for active vibro-acoustic control: Discussion and experiences. Control Engineering Practice 15 (2007) 163-176.

[4].S.M. Kuo, B.H. Lee, and W. Tian, Real-Time Digital Signal Processing: Implementations and Applications, 2nd Edition. John Wiley \& Sons, Ltd. ISBN: 0-470-01495-4. (2006).

[5].Zhi-cheng Qiu, Jian-da Han, Xian-min Zhang, Yue-chao Wang, Zhen-wei Wu, Active vibration control of a flexible beam using a non-collocated acceleration sensor and piezoelectric patch actuator, Journal of Sound and Vibration, 326(3-5): 438-455. (2009)

[6].P. Gardonio, Sensors and actuators for active structural acoustic and active vibration feedback control. IV ECCOMAS Thematic Conference on Smart Structures and Material, SMART'09. Porto, Portugal. (2009)

[7].Chinsuk Hong, Changjoo Shin and Weui Bong Jeong. ACCELERATION FEEDBACK CONTROL OF STRUCTURAL VIBRATION OF BEAMS WITH A MOMENT PAIR ACTUATOR. 18th International Congress on Sound and Vibration, 10-14 July 2011, Rio de Janeiro, Brazil. (2011). 


\section{Tables}

Table 1. Specifications of the two accelerometers

\begin{tabular}{lll}
\hline Specifications & Sensor 1 & Sensor 2 \\
\hline Measurement Range $(g)$ & \pm 5 & \pm 50 \\
\hline Sensitivity $(\mathrm{mV} / \mathrm{g})$ & 1000 & 100 \\
\hline Threshold $\left(\mathrm{ug}_{\mathrm{rms}}\right)$ & 120 & 1000 \\
\hline
\end{tabular}

\section{Caption of Figures}

Figure 1. Schematic of an active vibration control (AVC) system

Figure 2. Detectable acceleration range using a DSP and single-sensor system

Figure 3. Detectable acceleration range using a DSP and dual-sensor system

Figure 4. Flowchart of the dual-sensor detection system

Figure 5. Simulink Model of the Dual-Sensor Detection Component

Figure 6. (a) Original vibration signal from accelerometer; (b) measured signal by DSP using signal fusion of the Dual-Sensor detection component

Figure 7. Schematic of the active vibration control (AVC) system with dual-sensor feedback

Figure 8. SIMULINK model of active vibration control system with DSP-based dual-sensor acceleration detection and feedback

Figure 9. Comparison of plant vibration responses to a step disturbance. (From top to bottom: (a) The uncompensated plant; (b) with AVC using Single-Sensor Feedback of sensor 1; (c) with AVC using Single-Sensor Feedback of sensor 2; (d) with AVC using dual-sensor feedback.)

Figure 10. Comparison of plant vibration responses to a step disturbance: zoomed view. (From Top to Bottom: (a) The uncompensated plant; (b) with AVC using Single-Sensor Feedback of sensor 1; (c) with AVC using Single-Sensor Feedback of sensor 2; (d) with AVC using dual-sensor feedback.) 


\section{Figures}

Disturbance Forces

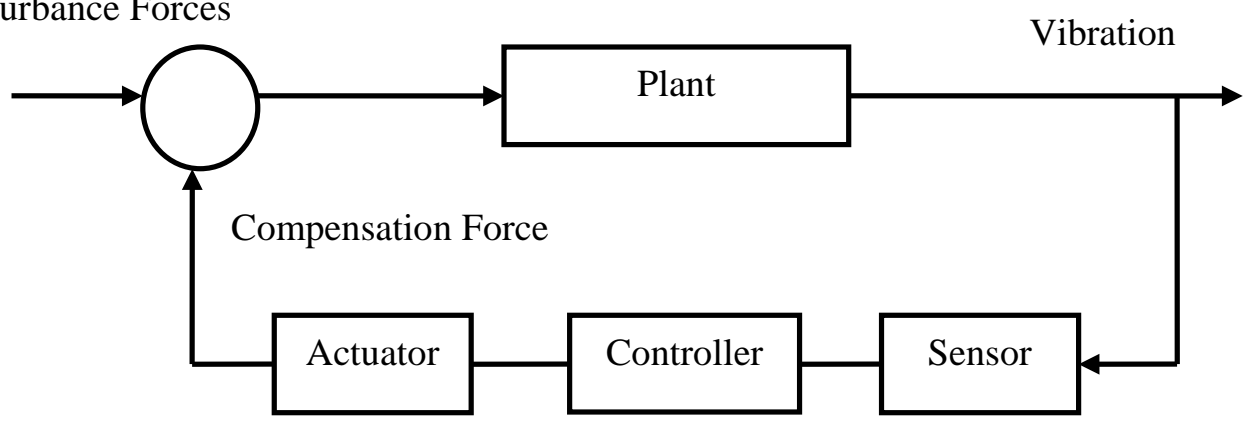

Figure 1. Schematic of an active vibration control (AVC) system

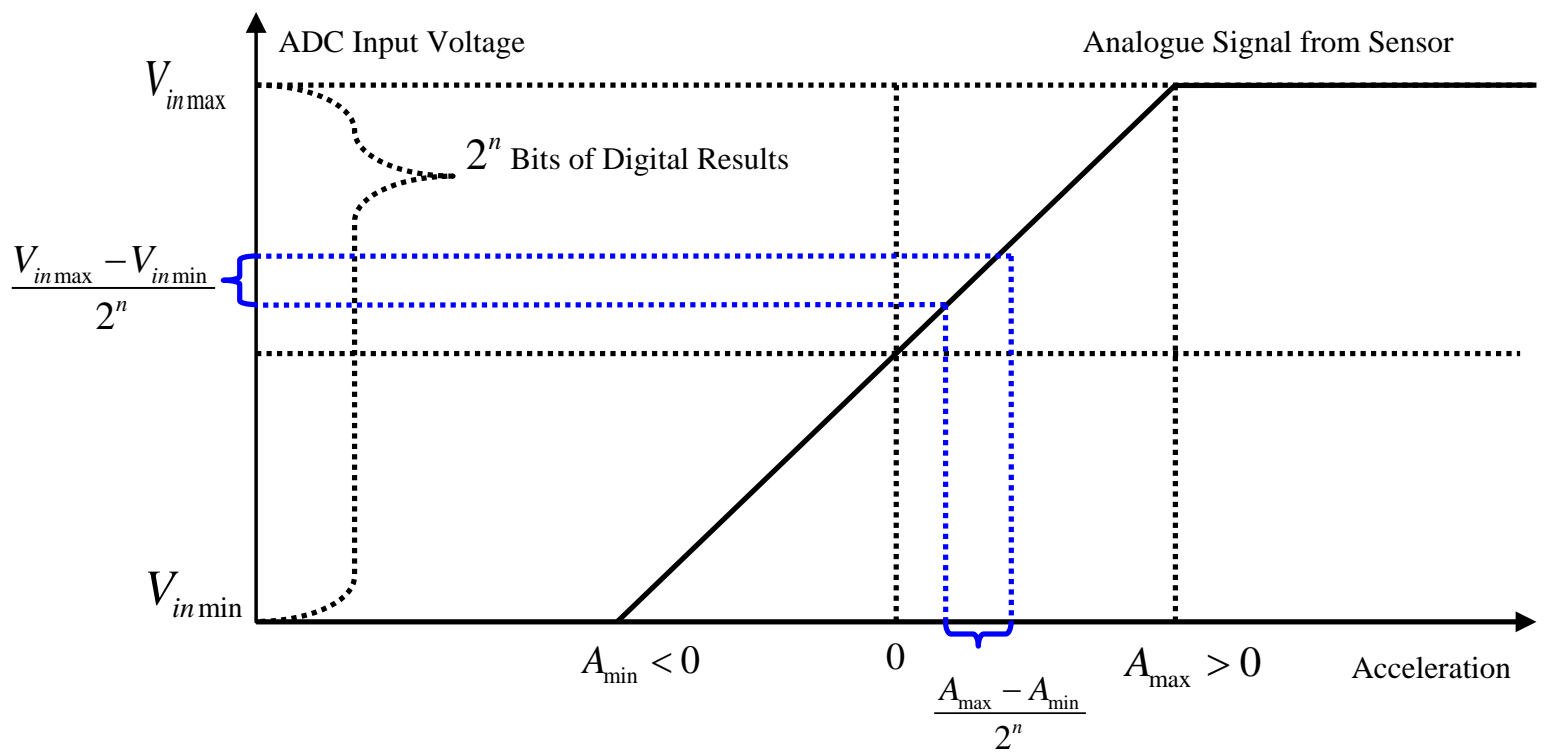

Figure 2. Detectable acceleration range using a DSP and single-sensor system 


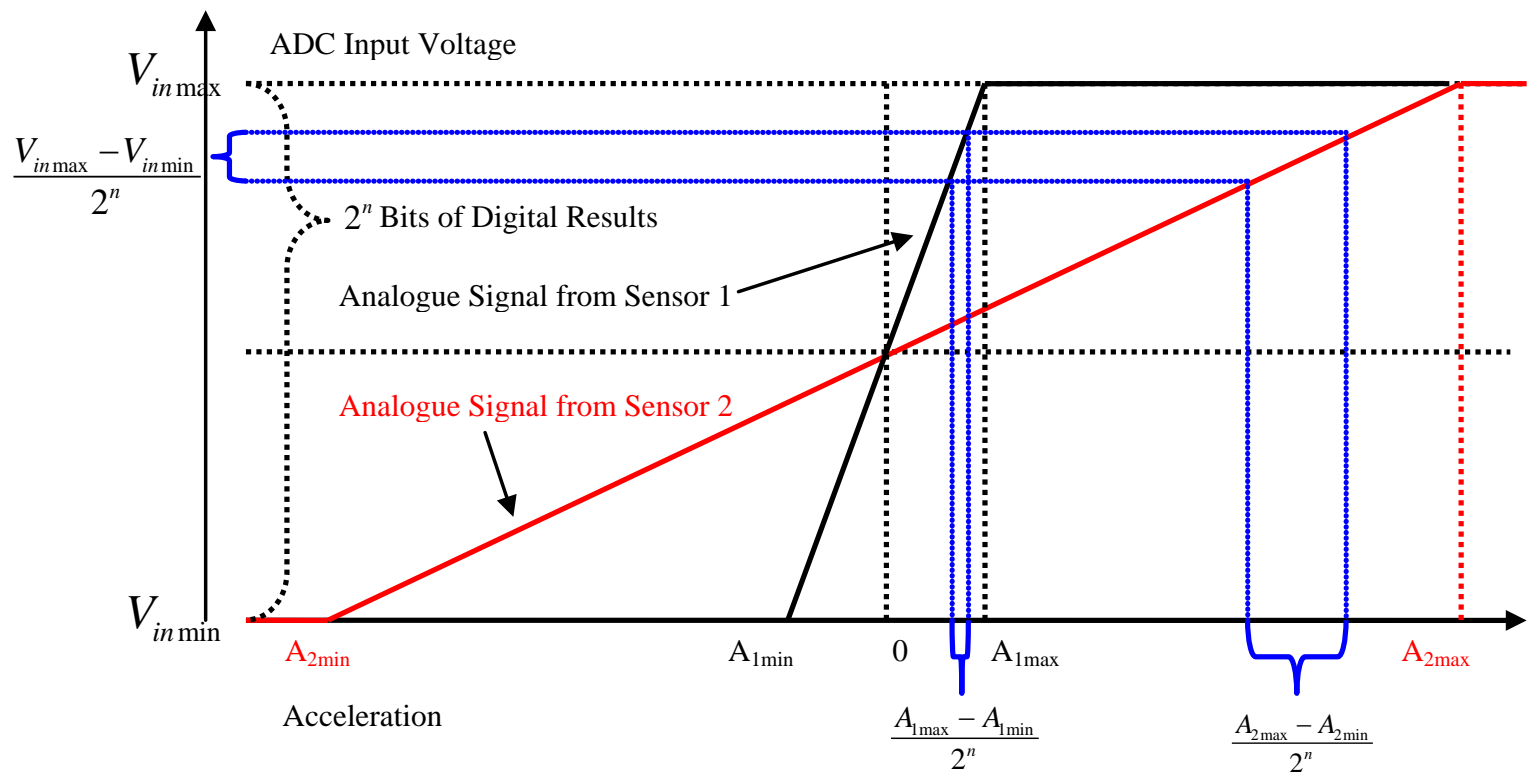

Figure 3. Detectable acceleration range using a DSP and dual-sensor system

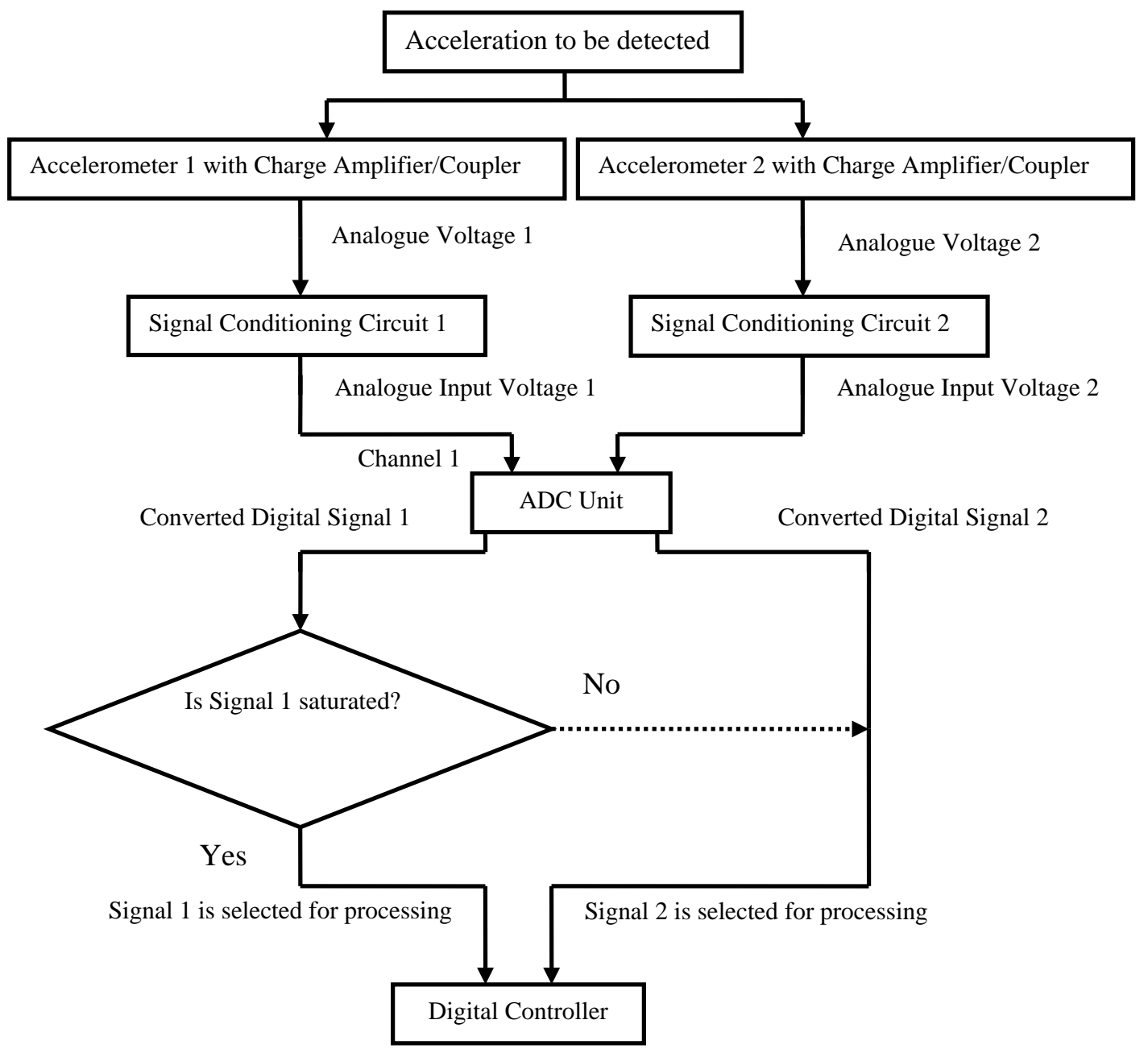

Figure 4. Flowchart of the dual-sensor detection system 


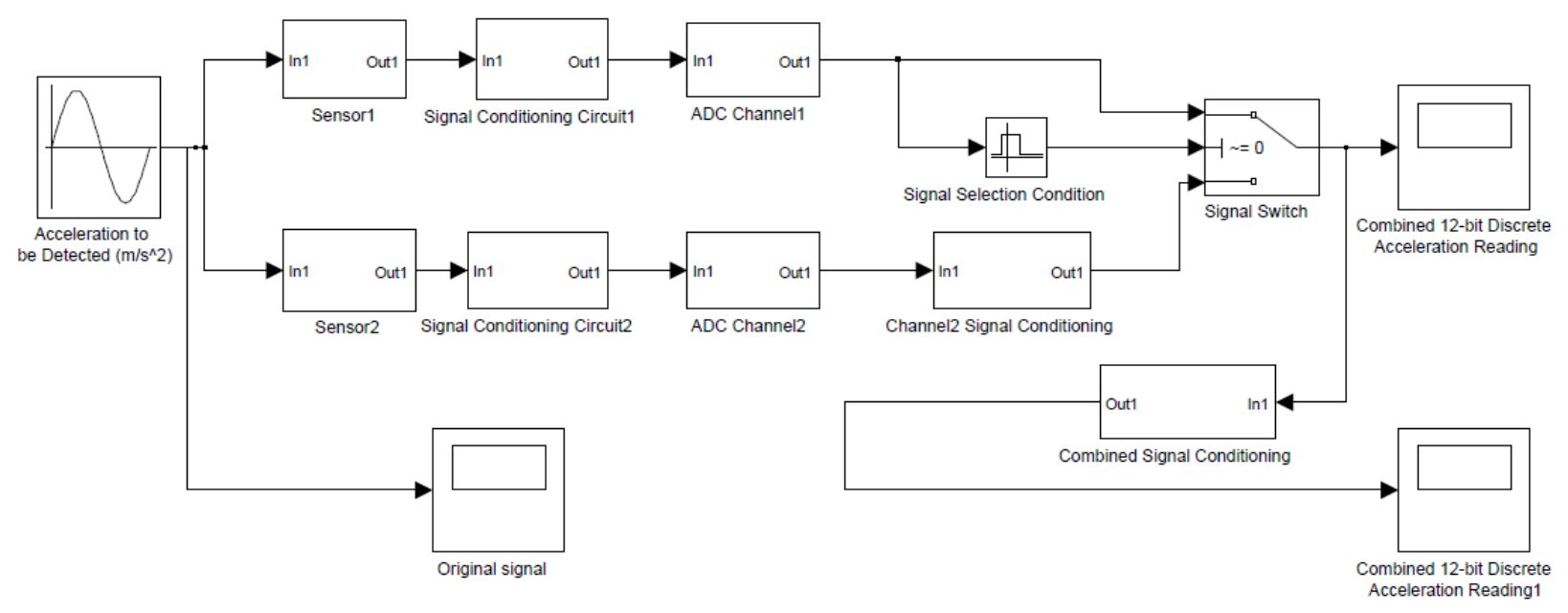

Figure 5. Simulink Model of the Dual-Sensor Detection Component 


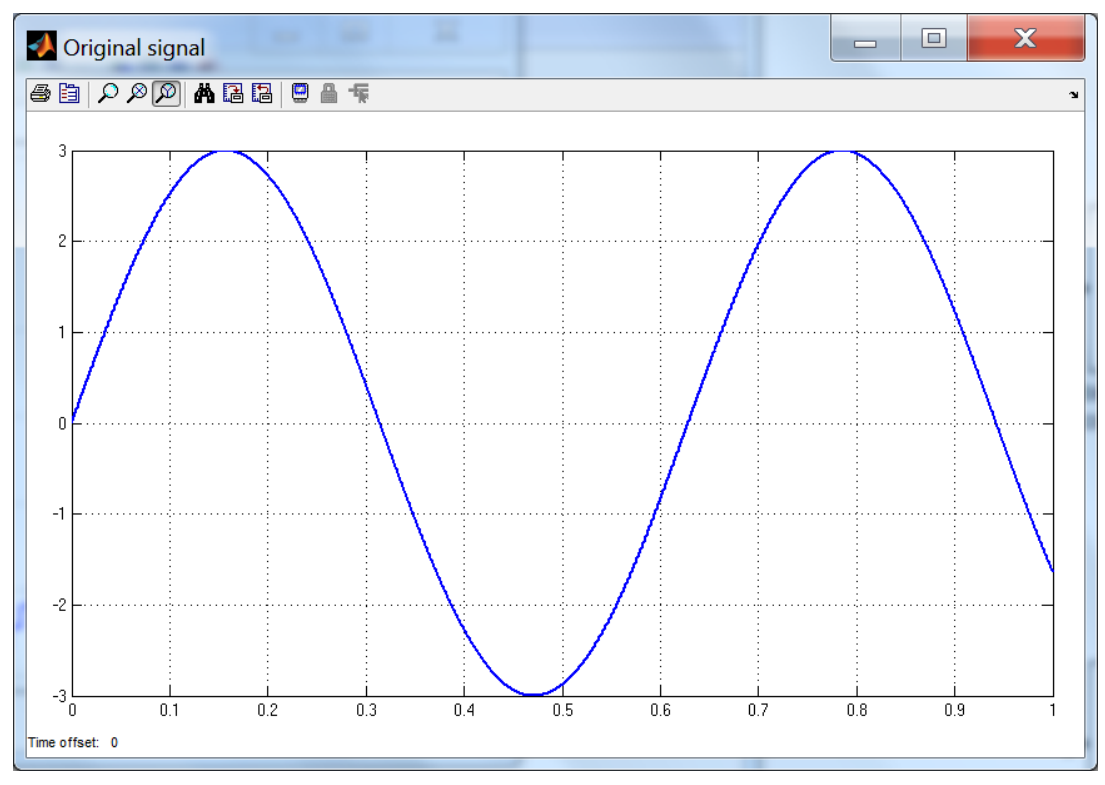

(a)

Combined 12-bit Discrete Acceleration Reading1

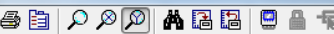

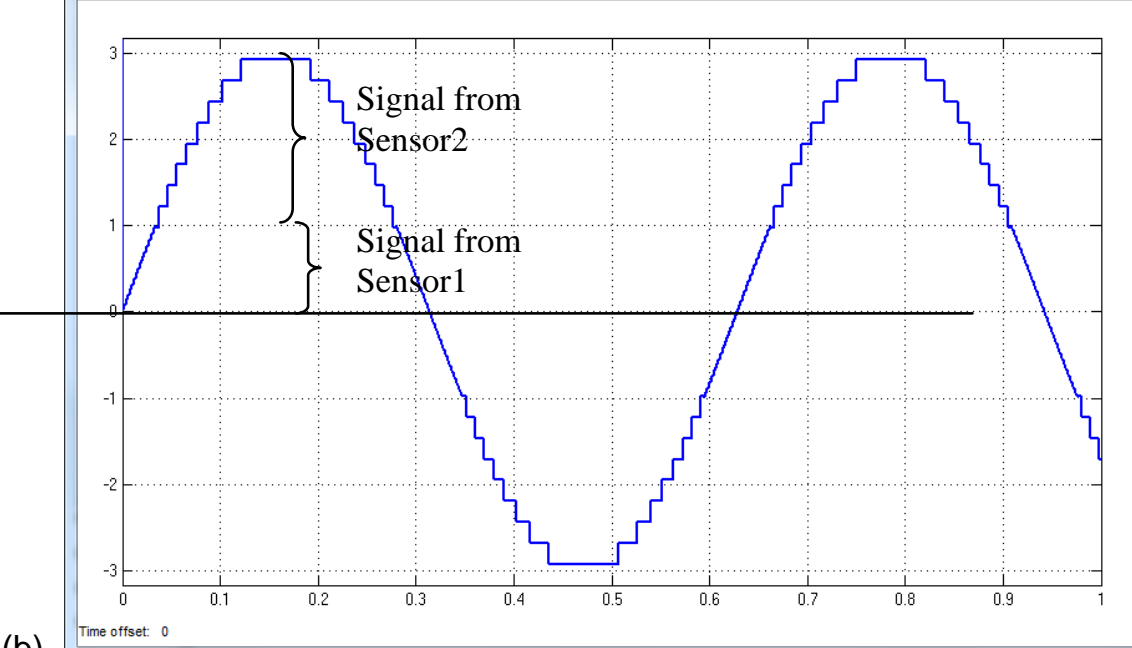

(b)

Figure 6. (a) Original vibration signal from accelerometer; (b) measured signal by DSP using signal fusion of the Dual-Sensor detection component 


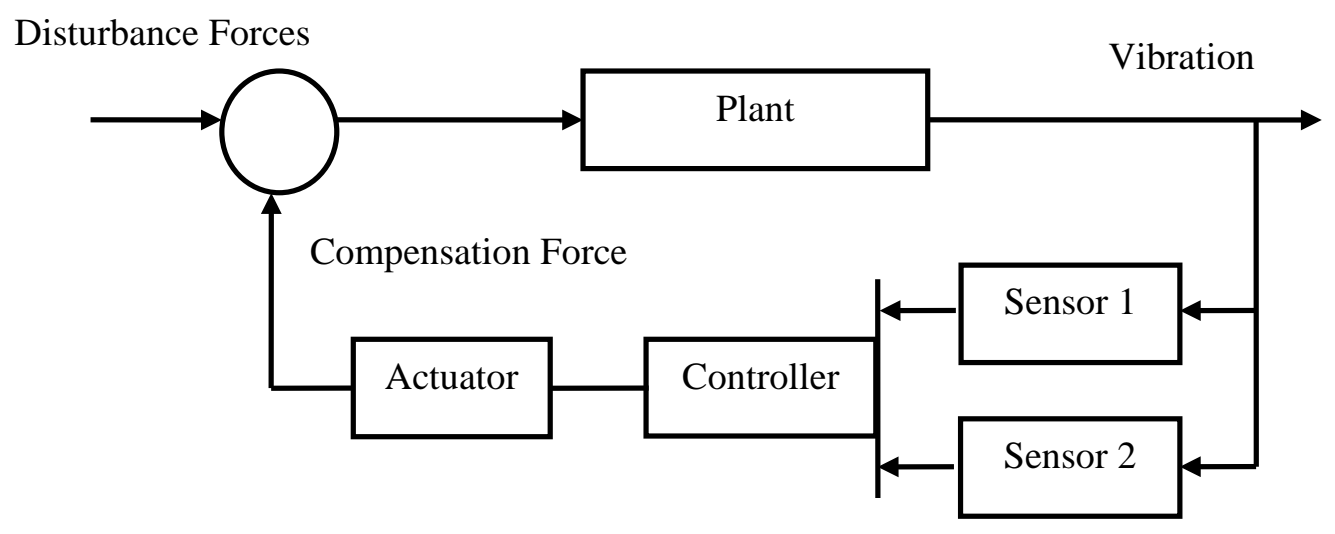

Figure 7. Schematic of the active vibration control (AVC) system with dual-sensor feedback

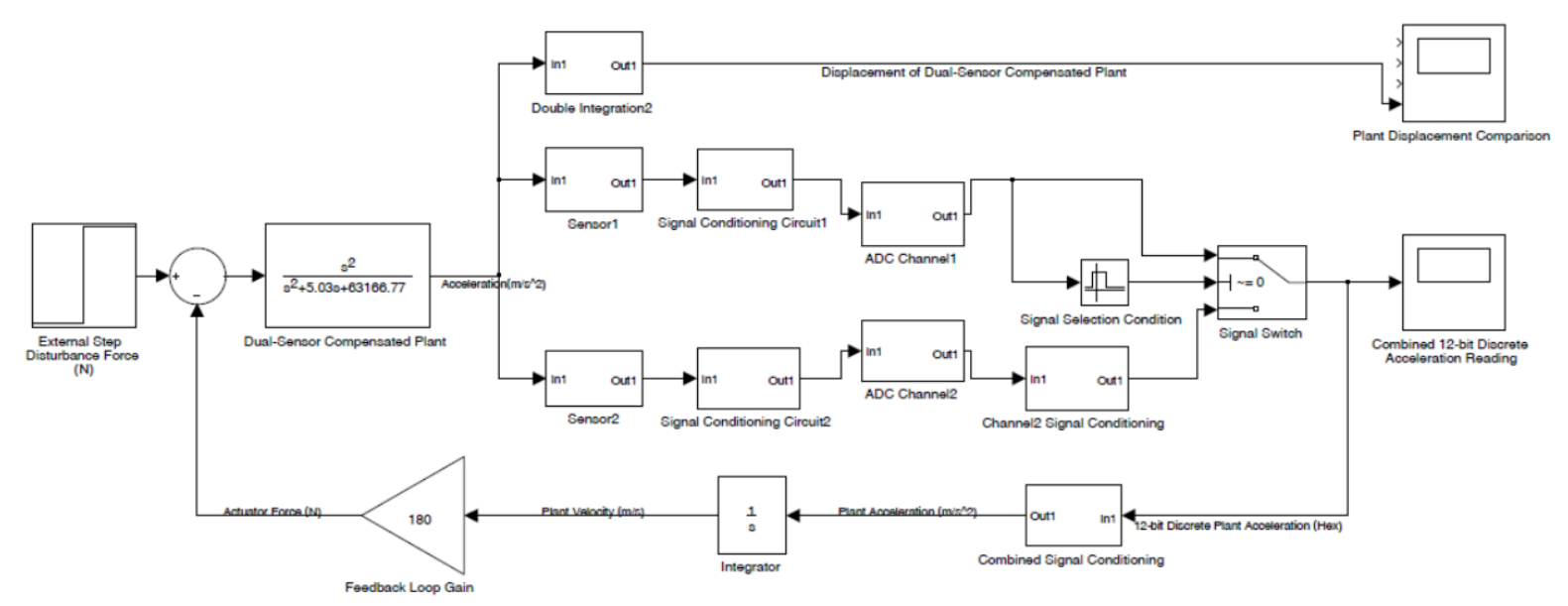

Figure 8. SIMULINK model of active vibration control system with DSP-based dual-sensor acceleration detection and feedback 


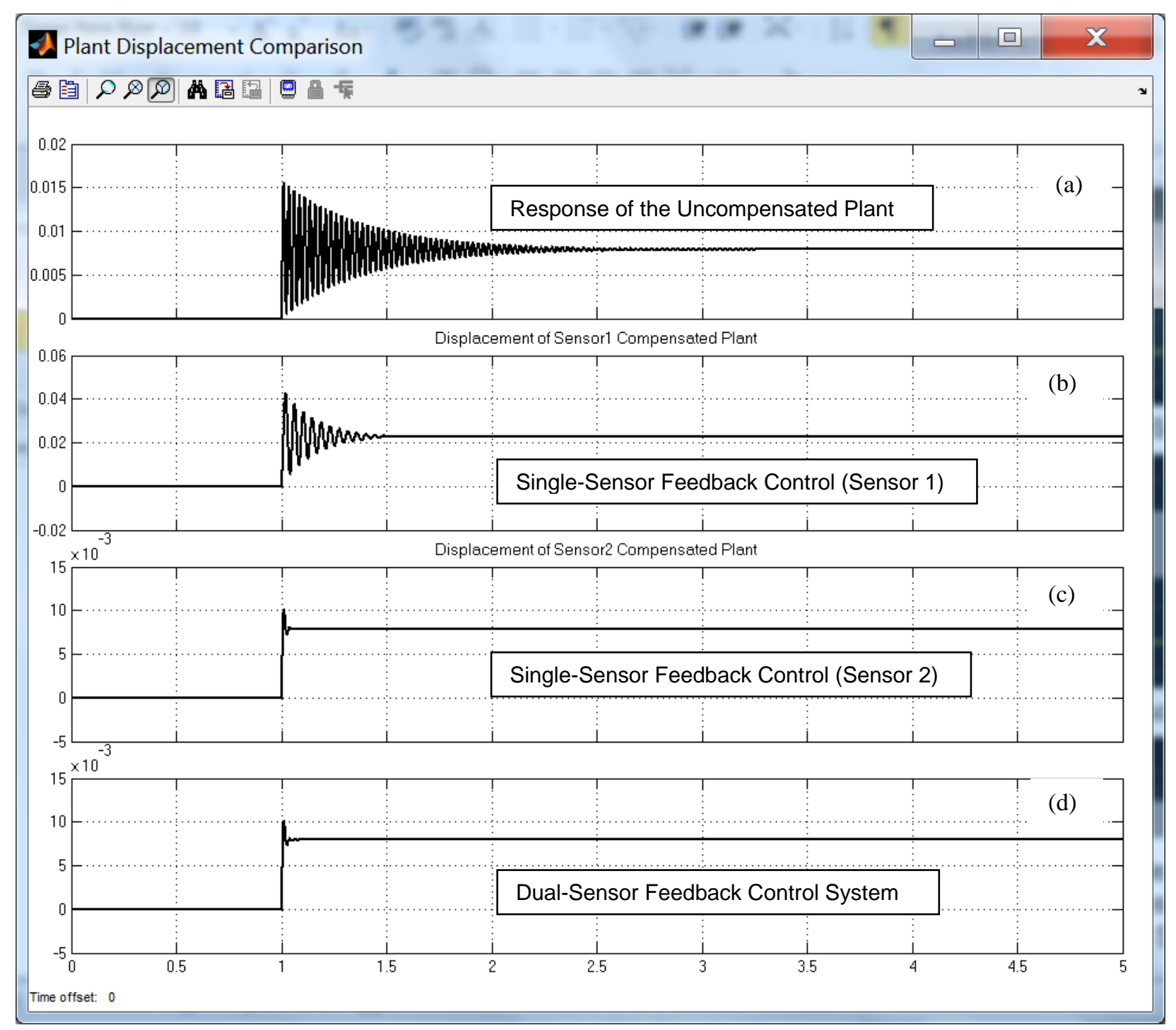

Figure 9. Comparison of plant vibration responses to a step disturbance. (From top to bottom: (a) The uncompensated plant; (b) with AVC using Single-Sensor Feedback of sensor 1; (c) with AVC using Single-Sensor Feedback of sensor 2; (d) with AVC using dual-sensor feedback.) 


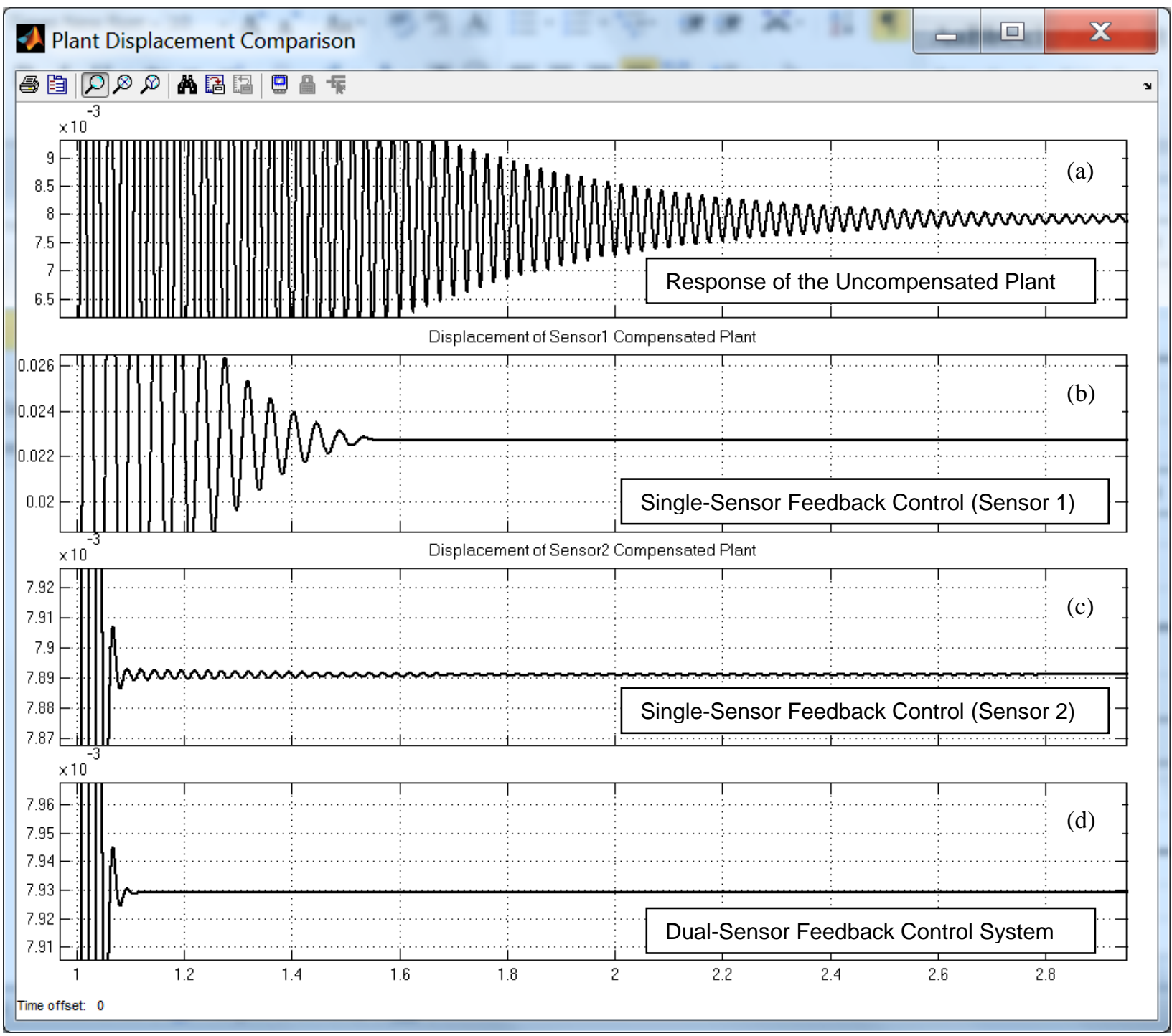

Figure 10. Comparison of plant vibration responses to a step disturbance: zoomed view. (From Top to Bottom: (a) The uncompensated plant; (b) with AVC using Single-Sensor Feedback of sensor 1; (c) with AVC using Single-Sensor Feedback of sensor 2; (d) with AVC using dual-sensor feedback.) 